\title{
A-Kinase Anchoring Proteins 10 Expression in Relation to 2073A/G Polymorphism and Tumor Progression in Patients with Colorectal Cancer
}

Mojin Wang, Dan Zhang, Rui Wang, Yuanyi Rui, Jin Zhou, Rong Wang, Bin Zhou, Xiaoran Huang, Lie Yang, Yuan Li, Jiankun Hu, Zongguang Zhou and Xiao-Feng Sun

\section{Linköping University Post Print}

\section{Tweet}

N.B.: When citing this work, cite the original article.

The original publication is available at www.springerlink.com:

Mojin Wang, Dan Zhang, Rui Wang, Yuanyi Rui, Jin Zhou, Rong Wang, Bin Zhou, Xiaoran Huang, Lie Yang, Yuan Li, Jiankun Hu, Zongguang Zhou and Xiao-Feng Sun, A-Kinase Anchoring Proteins 10 Expression in Relation to 2073A/G Polymorphism and Tumor Progression in Patients with Colorectal Cancer, 2013, Pathology and Oncology Research, (19), 3, 521-527.

http://dx.doi.org/10.1007/s12253-013-9612-6

Copyright: Springer Verlag (Germany) http://www.springerlink.com/?MUD=MP

Postprint available at: Linköping University Electronic Press http://urn.kb.se/resolve?urn=urn:nbn:se:liu:diva-97673 


\section{A-kinase anchoring proteins 10 expression in relation to 2073A/G polymorphism and tumor progression in patients with colorectal cancer}

MoJin Wang • Dan Zhang • Rui Wang • YuanYi Rui • Jin Zhou • Rong Wang • Bin Zhou • XiaoRan Huang • Lie Yang •Yuan Li • Jiankun Hu • ZongGuang Zhou • XiaoFeng Sun

M. Wang • D. Zhang • Y. Rui • J. Zhou • R. Wang • B. Zhou • L.Yang • J. Hu • Z. Zhou ( $\)$

Department of Gastrointestinal Surgery, Institute of Digestive Surgery, National Key Laboratory of Biotherapy, West China Hospital, Sichuan University, 37 Guo Xue Xiang, Chengdu 610041, China. e-mail: zhou767@163.com

R. Wang

Department of Gastroenterology, West China Hospital, Sichuan University, Chengdu, China

X. Huang

Department of Pathology, West China Hospital, Sichuan University, Chengdu, China

Y. Li

Department of Pediatric Surgery, Institute of Digestive Surgery and State Key Laboratory of Biotherapy, West China Hospital, Sichuan University, Chengdu, China

X. Sun

Division of Oncology, Department of Clinical and Experimental Medicine, Faculty of Health Sciences, University of Linköping, Linköping, Sweden 


\section{Abstract}

The cAMP/PKA signalling events regulated by A-kinase anchoring proteins 10 (AKAP10) is involved in tumorigenesis. Previous study showed that AKAP10 polymorphism (2073 A/G, I646V) was associated with colorectal cancer risk. However, there was no literature reporting the role of AKAP10 in the pathogenesis of colorectal cancer. The aim of the study was to investigate the clinicopathologic significance of A-kinase anchoring proteins 10 (AKAP 10) expression and the relationship with its polymorphism in colorectal cancer. The expression of AKAP10 was determined by immunohistochemical staining (IHC) and western blot assay on colorectal cancer (n=176), adenoma ( $n=87)$ and distant normal mucosa $(n=72) .176$ patients with colorectal cancer were genotyped for AKAP10 2073A/G polymorphism by TaqMan RT-PCR. We found that the positive expression rate of AKAP10 in colorectal cancer (59\%) was significantly higher than those in adenoma (39\%) and distant normal mucosa (42\%) $(P=0.004)$. There was no significant difference between adenoma and distant normal mucosa $(P=0.741)$. Positive AKAP10 staining was correlated with deeper tumor invasion $(P<0.001))$, lymph nodes metastasis $(P=0.022)$, advanced tumor stage $(P<0.001)$ and poorly differentiated degree $(P=0.003)$. Compared with AA genotype (52\%), positive expression of AKAP10 was significantly increased in colorectal cancer patients with the variant (AG+GG) genotypes (68\%, $P=0.033$ ). It was concluded that AKAP10 may play an important role in the development and progression of colorectal cancer.

Keywords: A-kinase anchoring proteins 10 • Immunohistochemical staining • Western blot Polymorphism • Colorectal cancer 


\section{Introduction}

Given the continually rising incidence, colorectal cancer has become a growing health problem and one of the leading diagnosed cancers in China. According to Vogelstein B's model, colorectal cancer has been regarded as a kind of genetic disease involved with multi-steps, multi-stages and multi-genes [1]. The cAMP-dependent protein kinase (PKA), one of the first discovered protein kinases, has been regarded as an essential element in colorectal carcinogenesis. The extracellular PKA in active is excreted by colorectal cancer cells. The up-regulation of PKA in the serum of cancer patients suggested that PKA served as a diagnostic and prognostic biomarker for colorectal cancer [2].

A-kinase anchoring proteins 10 (AKAP10), also known as dual specific AKAP2 (D-AKAP2), belongs to a family of structurally diverse but functionally related proteins having the ability of anchoring PKA and has the unique targeting sequences that compartmentalize the PKA-AKAP complex to specific subcellular locations. AKAP10 can bind to both RI and RII subunit isoforms of PKA, through an interaction with an N-terminal four-helix bundle (dimerization/ docking, D/D domain) in the R subunit dimer of PKA [3, 4]. The domain organization of AKAP10 is quite unique, containing two tandem, putative RGS domains, a PKA-binding motif, and a PDZ (PSD95/Dlg/ZO1)-binding motif [5-8]. These domains provide an element of spatial and temporal coordination between upstream cAMP/PKA signalling events and downstream AKAP-mediated signalling events.

The cAMP/PKA signalling events regulated by AKAP10 are involved in the control of cell proliferation, differentiation and death, which are crucial in tumorigenesis [9-11]. Thus, deregulation of the cAMP/PKA/AKAP10 signal pathway might result in colorectal carcinogenesis. Wirtenberger $\mathrm{M}$ et al. indicated that the patients who carried AKAP10 Val646 variant had a greater risk of developing familiar breast cancer [12]. The increased abilities of AKAP10 Val646 variant in binding and localizing PKA to its subcellular substrates maybe a disadvantage for the cell in carcinogenesis, resulting in a stronger mitogen effect of PKA. Based on this critical study, we have conducted a case-control study in a Southwestern Chinese population and found that AKAP10 polymorphism (2073 A/G, I646V) was associated with colorectal cancer risk. The Chinese individuals with the G genotype might be linked with the increased risk of developing colorectal cancer [13]. However, the AKAP10 expression in human colorectal cancer and its role in the carcinogenesis as well as the relationships to the clinicopathologic factors remain unclear. 
Therefore, we examined the expression of AKAP10 in primary colorectal cancer, distant normal mucosa, as well as adenoma, to determine whether AKAP10 expression is involved in tumor development, and whether the AKAP10 expression is correlated with its polymorphism. 


\section{Materials and methods}

\section{Patients and Samples}

The analyzed cohort of patients underwent primary surgery for colorectal cancer at West China Hospital, Chengdu, from May 2006 to September 2007. These patients did not receive any chemotherapy and radiotherapy before operation. Adenoma samples were harvested from patients with colorectal adenoma during the colonoscopy examination at endoscope centre of West China Hospital, Chengdu. All the excised tissues were placed immediately in liquid nitrogen and stored at $-80^{\circ} \mathrm{C}$ until analysis. The whole samples were examined for the presence of cancer and adenoma cells at the department of pathology by two skilful pathologists in West China Hospital. The information of gender, age, tumor site, growth pattern, invasive depth, lymph nodes metastasis and differentiation in colorectal cancer patients were obtained from surgical and pathological records. The growth pattern was based on the pattern of growth and invasiveness. Differentiation was graded as well, moderately and poorly differentiated according to the WHO criteria. Clinical and pathological stages were defined according to the TNM stages [14]. For immunohistochemical analysis, the staining was performed on primary cancer $(n=176)$, adenoma $(n=87)$ and distant normal mucosa $(n=72)$. The distant normal mucosa samples were taken from the margin of distant resection, and were histologically free of pre-cancer and cancer. For genotyping, the genomic DNA was isolated from venous blood of patients with colorectal cancer. The study was approved by the SiChuan University Ethics Committee and written informed consent was obtained from all the participants.

Immunohistochemical analysis

Five-micrometer paraffin-embedded sections were deparaffinized in xylene and rehydrated in descending concentrations of ethanol and finally in double distilled $\mathrm{H}_{2} \mathrm{O}$. To expose masked epitopes, the sections were cooked at high pressure with Tris-EDTA buffer (pH9.0) for 5 minutes. After rinsed thrice in phosphate-buffered saline (PBS, pH7.4), the endogenous peroxidase activity was suppressed by $0.3 \%$ hydrogen peroxide in methanol for $15 \mathrm{~min}$ and then the sections were washed three times in PBS. The sections were treated with $2 \%$ normal goat serum for 30 min to block nonspecific background staining, and then incubated with primary mouse monoclonal AKAP10 antibody (Santa Cruz Biotechnology, USA) at a dilution of 1:50 in a humidified chamber at $4^{\circ} \mathrm{C}$ over night. 
Subsequently, the sections were incubated with a biotinylated goat antimouse IgG (ZYMED, USA) for 40 min, followed by horseradish peroxidase-streptavidin complex (S-A/HRP; ZYMED) for an additional $30 \mathrm{~min}$, diluted as recommended by the manufacturer. DAB was used as the chromogen, with hematoxylin as the counterstain. The matched normal mucosa and primary tumors were stained in the same run of the immunostaining to avoid bias on the pattern and intensity of staining. Sections known to show strong immunostaining for AKAP10 were included in each run receiving either primary antibody or PBS, as positive and negative controls. In all of the staining procedures, the positive controls showed staining clearly, and there was no staining in the negative controls.

Stained sections were examined microscopically and scored independently by two investigators (MJ Wang and XR Huang) in a blinded fashion without clinicopathological or biological information. Patterns, cellular localization, staining intensity and percentage of AKAP10 positive cells were recorded. The evaluation of AKAP10 expression was performed using an immunoreactive score (IRS) system, according to which IRS= SI (staining intensity) $\times$ PP (percentage of positive cells) [15]. SI of tumor cells was scored as 0 (negative staining), 1 (faint yellow staining), 2 (brown staining), and 3 (dark brown staining). PP was scored as 0 (no positive cells), 1 ( $<10 \%$ positive cells), 2 (10 50\% positive cells), and 3 ( $>50 \%$ positive cells). A total of 10 high-power visual fields with 100 cells per field, counted from different areas of each section were chosen at random for IRS evaluation followed by the calculation of the average IRS. The final intensity of AKAP10 staining was defined as negative, weak, moderate and strong, corresponding to IRS values of $\leq 1,<4,<6$ and $\geq 6$, respectively. In the cases with discrepant scoring, a consensus score was reached after re-examination. To avoid artificial effect, cells in areas with necrosis, poor morphology or in the margins of sections were not counted.

\section{Western blot assay}

Total protein from primary colorectal cancer, adenoma and distant normal mucosa samples was extracted with RIPA lysis buffer (KeyGEN Biotech., China) and quantified using BCA Protein Assay Kit (Thermo Scientific, USA). After denaturation at $100^{\circ} \mathrm{C}$ for 5 min, equal amounts of proteins $(20 \mu \mathrm{g})$ were loaded into each well of a 10\% SDSPAGE gel. After electrophoresis, the proteins were transferred onto a $0.2 \mathrm{~mm}$ polyvinylidene difluoride membrane at $100 \mathrm{~V}$ for $90 \mathrm{~min}$ (Bio-Rad, USA). After completing protein transfer, the membrane was blocked in 5\% (w/v) skimmed milk in PBS and incubated overnight with mouse monoclonal antibody against AKAP10 (diluted 1:500; Santa Cruz 
Biotechnology, USA) or rabbit anti-human $\beta$-actin (diluted 1:1,000; Cell Signaling Technology, USA). After washing three times (10 min per time) with TBST (Trisbuffered saline with $0.1 \%$ Tween-20) buffer, the membrane was incubated with secondary horseradish peroxidaseconjugated anti-mouse antibodies (diluted 1:3,000; Santa Cruz Biotechnology, USA) for 2 h at room temperature, developed with an enhanced chemiluminescence and visualized with a Multimage Light Cabinet (Alpha Innotech Corporation, USA).

\section{DNA extraction}

The genomic DNA was isolated from peripheral blood of 176 colorectal cancer patients using proteinase $\mathrm{K}$ digestion, followed by phenol-chloroform extraction and ethanol precipitation. The concentration and purity of the DNA were measured with a spectrophotometer.

\section{Genotyping}

The TaqMan RT-PCR targeted for AKAP10 A-2073G polymorphism was performed according to previous description [13]. Primers and TaqMan probes were purchased from Invitrogen Biotechnology Co. Ltd (Carlsbad, CA. USA): forward 5'-GGAAGAGCTAGCTTGGAA-3', reverse 5'-TAGATTTCTCTAACGGTTGAT-3', $\quad$ FAM-probe 5‘-FAM-AGCCTGCTGCATAATGTCACTGA(TAMRA)-3’, HEX-probe 5‘-HEX-AGCCTGCTGCATAACGTCACTGA(TAMRA)-3’. PCR amplification was carried out in a total volume of $30 \mu \mathrm{l}$ reaction mixture containing $1 \mu \mathrm{l}(\sim 5$ nanograms $)$ genomic DNA, $1 \times$ PCR buffer (Takara, Dalian, China), $2.5 \mathrm{mM} \mathrm{MgCl}_{2}$ (Takara), $0.3 \mathrm{mM}$ dNTP, $0.17 \mathrm{mM}$ of each primer, $5 \times 10^{-5}$ U/ml rTaq DNA polymerase (Takara) and $\mathrm{H}_{2} \mathrm{O}$. Conditions for the PCR reaction were a 5-min initial denaturation at $94^{\circ} \mathrm{C}$ followed by 40 times of amplification at $94^{\circ} \mathrm{C}$ for $20 \mathrm{~s}, 58^{\circ} \mathrm{C}$ for $40 \mathrm{~s}$ on iCycler iQ real-time PCR detection System (Bio-Rad). Approximately 10\% of the samples were randomly selected for repeated assays. All rare homozygous genotypes (GG) were confirmed by direct DNA sequencing, and the results were $100 \%$ concordant.

\section{Statistical analysis}

The SPSS 11.5 software package (Chicago, IL) was used for all statistical analyses. Chi-squared test was used to test the difference of AKAP10 expression among primary cancer, adenoma and distant 
normal mucosa. The relationship between the AKAP10 expression and clinicopathological factors/ genotypes was also analyzed by chi-squared test. Calculations for Hardy-Weinberg equilibrium (HWE) were carried out using the Hardy-Weinberg equilibrium tool offered by the Institute of Human

Genetics, Technische Universita“t, Munich, Germany (http:// ihg.gsf.de/cgi-bin/hw/hwa1.pl). All $P$-values cited were two-sided and $P$-values $<0.05$ were judged as statistically significant. 


\section{Results}

AKAP10 expression among primary cancer, adenoma and distant normal mucosa

By immunohistochemistry (IHC), the protein expression and subcellular localization of AKAP10 were determined in primary cancer, adenoma and distant normal mucosa. No significant differences with respect to clinicopathological characteristics were found among negative, weak, moderate and strong staining. Therefore, for further analyses the former group was classified as negative, and the latter three groups were classified as positive in our study. In the positive tissues, the brown particles were distributed in the cytoplasm and nucleus of normal, adenoma and cancer cells. But in the negative tissues and stromal components, these yellow or brown particles could not be visualized (Fig. 1). The positive expressions of AKAP10 in the primary cancer, adenoma and distant normal mucosa were 59, 39 and $42 \%$, with significant difference $(P=0.004)$. The comparisons of either distant normal mucosa versus primary cancer or adenoma versus primary cancer were statistical significant with the $P$ value of 0.016 and 0.003 , respectively. Additionally, the comparison of normal tissues versus adenoma had no statistical difference $(P=0.741)$ (Table 1$)$.

By Western blot assay, the specificity of the AKAP10 antibody used in IHC was examined. The quantitative analysis showed that the expression of AKAP10 was abundantly increased in the primary cancer than adenoma or distant normal mucosa $(P=0.021$, Figure 2$)$. This alteration was in accordance with that of the IHC staining.

AKAP10 expression in primary cancer in relation to clinicopathological characteristics

The association of AKAP10 expression in the primary cancer with clinicopathological characteristics was presented in Table 2. Using stratified analysis, we found that positive expression of AKAP10 was correlated with the invasive depth of cancer $(P<0.001)$, lymph nodes metastasis $(P=0.022)$, TNM stage $(P<0.001)$ and the grade of cancer differentiation $(P=0.003)$. There was no correlation of AKAP10 expression with gender, age, tumor site and growth pattern. 
The relationship between AKAP10 expression and polymorphism

The distribution of the genotypes of AKAP10 polymorphism in colorectal cancer was analyzed by Hardy-Weinberg equilibrium test, and results demonstrated $P>0.05$. In 176 colorectal cancer patients, we analyzed the AKAP10 expression and the genotypes of the polymorphism and observed that 54 (52\%) colorectal cancer patients with the AA genotype exhibited AKAP10-positive characteristics, 15 (63\%) in AG genotype, and 34 (71\%) in GG genotype. There was a higher trend that the colorectal cancer patients with the GG genotype had stronger staining compared to AA or AG genotype, although the difference was not statistically significant ( $P=0.081$, data not shown). We further combined AG and GG genotypes as variant genotypes. The positive expression of AKAP10 had been found significantly increased in colorectal cancer patients with the variant genotypes (49/72, 68\%), compared with AA genotype (54/104, 52\%, $P=0.033$, Figure 3). The stratification analysis showed the association between the AKAP10 expression and polymorphism was not statistically significant in the subgroups which were divided according to invasive depth, lymph nodes metastasis, TNM stage and differentiation $(P>0.05)$. 


\section{Discussion}

A-kinase anchoring proteins are a family of anchoring proteins that, although being structurally diverse, have the ability to bind to and target PKA in common [16]. As a representative of dual-specificity AKAPs, AKAP10 has the conservative domain to bind to both RI and RII subunit isoforms of PKA, and tethers the cAMP /PKA signalling complex to distinct subcellular locations. It has been proved that cAMP/PKA signal pathway is implicated in the control of cell proliferation, differentiation and death, therefore, has played a key role in the carcinogenesis $[10,11]$. The overexpression of PKA-RI $\alpha$ has been observed in several tumors, including colorectal cancer [17, 18]. Cho et al. [2] reported the up-regulation of extracellular PKA in colon carcinoma cells, and suggested PKA served as a diagnostic and prognostic biomarker for colorectal cancer [19, 20]. The studies of 787 familial breast cancer patients and 993 controls showed that the Ile646Val polymorphism of AKAP10 was associated with an increased familial breast cancer risk [12]. In a previous case-control study, we firstly found that the Ile646Val polymorphism of AKAP10 was significantly associated with colorectal cancer risk [13].

In the present study, we found that AKAP10 was highly expressed in primary cancer compared with adenoma or distant normal mucosa. No significant difference in AKAP10 expression, however, was detected between adenoma and distant normal mucosa. For our limited knowledge, this was the first study reporting the AKAP10 expression in colorectal cancer. The appearance of strong AKAP10 expression in cancer with poor differentiation was much more frequent than in that with better differentiation. High level of AKAP10 expression in poor differentiated colorectal cancer was in accordance with the fact that dysregulation of cAMP/PKA signaling would distort the balance of cell growth and differentiation. Although there was the controversy about the role of PKA in cell differentiation, several researchers attributed this phenomenon to PKA-I/-II which exerted different, even reverse functions, through regulating a cluster of differentiation genes [9, 21, 22]. Our results suggested that the AKAP10 may selectively anchor specific subtype of PKA in colorectal cell and play a role in the differentiation of colorectal cancer. In addition, given the tumor invasion, metastasis in regional lymph nodes and especially TNM stage have been thought as the powerful predictive index to prognosis in colorectal cancer, closely linked high expression of AKAP10 to deeper tumor invasion, positive lymph nodes and advanced tumor stage detected in our results indicated that AKAP10 not only played an essential role in the development and progression of colorectal cancer, but also served as a biomarker to predict the prognosis of the patients with colorectal cancer. 
Furthermore, we demonstrated that there was a significant association between AKAP10 expression and the Ile646Val polymorphism of AKAP10 in colorectal cancer. Compared with wild type (AA), expression of AKAP10 in colorectal cancer with the variant genotypes (AG+GG) was significantly higher. As described above, we previously showed that this variant (G) was associated with the increased risk of colorectal cancer. The Ile646Val polymorphism of AKAP10 located in the PKA binding domain have been found in several types of diseases [12, 23, 24]. This variation has been thought to impact the binding to PKA in an isoform-specific manner both in vitro and in vivo. The researchers further pointed out that the Val variant had stronger ability to anchor the PKA-RI $\alpha$ than the Ile variant, resulting in alterations in the subcellular distribution of the recombinantly expressed PKA-RI $\alpha$ [25]. Besides the increasing combining power at PKA-RI $\alpha$, the Val variant may participate in cAMP/PKA/AKAP10 signaling complex conveying mitogenic signals through up-regulation of AKAP10. In addition, the stratification analysis showed the association between the AKAP10 expression and polymorphism was not statistically significant in the subgroups which were divided according to invasive depth, lymph nodes metastasis, TNM stage and differentiation. There may be different roles of AKAP10 involved in developmental stages of colorectal carcinogenesis. For incomplete records of prognosis, we have not analyzed the relationship between AKAP10 polymorphism and the survival of colorectal cancer patients in this study. Therefore, the precise mechanisms underlying the relationship of cAMP/PKA/ AKAP10 signaling and AKAP10 polymorphism with colorectal carcinogenesis need further investigation in the future.

In conclusion, the expression of AKAP10 in colorectal cancer was higher than that in distal normal colorectal tissues and adenoma. Increased AKAP10 expression in primary colorectal cancer was related to poor differentiation, deeper tumor invasion, positive lymph nodes metastasis and advanced tumor stage. Compared with wild type, the positive expression of AKAP10 was significantly increased in colorectal cancer patients with the variant genotypes. Therefore, our findings suggest that AKAP10 expression may play an important role in the development and progression of colorectal cancer. However, these findings may be affected by the number of histological samples and the method only at protein level, thus need confirmation in a deeply and larger scale research.

\section{Acknowledgments}

We thank Professors Zhou and Sun for guiding this study, the members of Digestive Surgery Institution 
of West China Hospital for useful assistance and colleagues of the Department of Gastrointestinal Surgery for providing experimental specimens. This study was supported by the National Natural Science Founding of China (No. 30830100). 


\section{References}

1 Vogelstein B, Fearon ER, Hamilton SR, et al (1988) Genetic alterations during colorectal-tumor development. N Engl J Med 319: 525-532

2 Cho YS, Park YG, Lee YN, et al (2000) Extracellular protein kinase A as a cancer biomarker: its expression by tumor cells and reversal by a myristatelacking $\mathrm{Ca}$ and RII $\beta$ subunit overexpression. Proc Natl Acad Sci USA 97: 835-840

3 Wong W, Scott JD (2004) AKAP signalling complexes: focal points in space and time. Nat Rev Mol Cell Biol 5: 959-970

4 Huang L, Durick K, Weiner JA, Chun J, Taylor SS (1997) D-AKAP2, a novel protein kinase A anchoring protein with a putative RGS domain. Proc Natl Acad Sci USA 94: 11184-11189

5 Hamuro Y, Burns L, Canaves J, Hoffman R, Taylor SS, Woods V (2002) Domain organization of D-AKAP2 revealed by enhanced deuterium exchange-mass spectrometry (DXMS). J Mol Biol 321: 703-714

6 Sheng M, Sala C (2001) PDZ domains and the organization of supramolecular complexes. Annu Rev Neurosci 24: 1-29

7 Wang L, Sunahara RK, Krumins A, et al (2001) Cloning and mitochondrial localization of full-length D-AKAP2, a protein kinase A anchoring protein. Proc Natl Acad Sci USA 98: 3220-3225

8 Burns-Hamuro LL, Barraclough DM, Taylor SS (2004) Identification and functional analysis of dual-specific A kinase-anchoring protein-2. Methods Enzymol 390: 354-374

9 Neary CL, Nesterova M, Cho YS, Cheadle C, Becker KG, Cho-Chung YS (2004) Protein kinase A isozyme switching: eliciting differential cAMP signaling and tumor reversion. Oncogene $23: 8847-8856$

10 Cross TG, Scheel-Toellner D, Henriquez NV, Deacon E, Salmon M, Lord JM (2000) Serine/threonine protein kinases and apoptosis. Exp Cell Res 256: 34-41

11 Tasken K, Skalhegg BS, Tasken KA et al (1997) Structure, function, and regulation of human cAMP-dependent protein kinases. Adv Second Messenger Phosphoprotein Res 31: 191-204

12 Wirtenberger M, Schmutzhard J, Hemminki1 K et al (2007) The functional genetic variant Ile646Val located in the kinase binding domain of the A-kinase anchoring protein 10 is associated with familial breast cancer. Carcinogenesis 28: 423-426

13 Wang MJ, Zhou ZG, Wang L, et al (2009) The Ile646Val (2073A>G) Polymorphism in the Kinase-Binding Domain of A-Kinase Anchoring Protein 10 and the Risk of Colorectal Cancer. Oncology 76: 199-204

14 Compton CC, Greene FL (2004) The Staging of Colorectal Cancer: 2004 and Beyond. CA Cancer J Clin 54: 295-308.।

15 Yang L, Zhang H, Zhou ZG, Yan H, Adell G, and Sun XF (2011) Biological Function and Prognostic Significance of Peroxisome Proliferator-Activated Receptor $\delta$ in Rectal Cancer. Clin Cancer Res 17: 3760-3770

16 Dodge-Kafka KL, Soughayer J, Pare GC, et al (2005) The protein kinase A anchoring protein mAKAP coordinates two integrated cAMP effector pathways. Nature 437: 574-578

17 Bradbury AW, Carter DC, Miller WR, Cho-Chung YS, Clair T (1994) Protein kinase A (PKA) regulatory subunit expression in colorectal cancer and related mucosa. Br J Cancer 69: 738-742

18 Bold RJ, Alpard S, Ishizuka J, Townsend CM Jr, Thompson JC (1994) Growth-regulatory effect of gastrin on human colon cancer cell lines is determined by protein kinase a isoform content. Regul 
Pept 53: 61-70

19 Nesterova MV, Johnson N, Cheadle C, et al (2006) Autoantibody cancer biomarker: extracellular protein kinase A. Cancer Res 66: 8971-8974

20 Hensley HH, Hannoun-Levi JM, Hachem P, et al (2011) PKA knockdown enhances cell killing in response to radiation and androgen deprivation. Int J Cancer 128: 962-973

21 Chen TC, Hinton DR, Zidovetzki R, Hofman FM (1998) Upregulation of the cAMP/PKA pathway inhibits proliferation, induces differentiation, and leads to apoptosis in malignant gliomas. Lab Invest 78: $165-174$

22 Siddappa R, Mulder W, Steeghs I, et al (2009) cAMP/PKA signaling inhibits osteogenic differentiation and bone formation in rodent models. Tissue Eng Part A 15: 2135-2143

23 Loniewska B, Clark JS, Kaczmarczyk M, et al (2012) Possible counter effect in newborns of 1936A $>$ G (I646V) polymorphism in the AKAP10 gene encoding A-kinase-anchoring protein 10. J Perinatol 32: 230-234

24 Zukowski M, Bohatyrewicz R, Biernawska J, et al (2009) Association of the A1936G (rs203462) of A-kinase anchoring protein 10 polymorphisms with QT interval prolongation during kidney transplantation. Transplant Proc 41: 3036-3038

25 Kammerer S, Burns-Hamuro LL, Ma Y, et al (2003) Amino acid variant in the kinase binding domain of dual-specific A kinase-anchoring protein 2: a disease susceptibility polymorphism. Proc Natl Acad Sci USA 100: 4066-4071 
Table 1 The result of immunohistochemical staining for AKAP10 in colorectal distant normal mucosa, adenoma and carcinoma

\begin{tabular}{lccccc}
\hline & & \multicolumn{3}{c}{ Staining } & \\
\cline { 3 - 4 } & Total & Negative (\%) & Positive (\%) & \\
\cline { 3 - 5 } Distant normal mucosa & 72 & $42(58)$ & $30(42)$ & \\
Adenoma & 87 & $53(61)$ & $34(39)$ & & \\
Carcinoma & 176 & $73(41)$ & $103(59)$ & $\chi^{2}=11.26$ & $P=0.004$ \\
\hline
\end{tabular}

The comparison of distant normal mucosa versus adenoma: $\chi^{2}=0.11 \quad(P=0.741)$; distant normal mucosa versus carcinoma: $\chi^{2}=5.838(P=0.016)$; adenoma versus carcinoma: $\chi^{2}=8.818(P=0.003)$ 
Table 2 The relationship between the results of AKAP10 immunohistochemical staining and the clinicopathologic characteristics in colorectal cancer patients

\begin{tabular}{lcc}
\hline \multirow{2}{*}{ Characteristics } & \multicolumn{2}{c}{ Staining } \\
\cline { 2 - 3 } & Negative (\%) & Positive (\%) \\
\hline
\end{tabular}

Gender

Male

Female

Age (years)

$\leq 58$

$>58$

Tumor site

Colon

Rectum

Invasive depth

Mucosa or submucosa

Proper muscle

Serosa or penetrate the serosa

Lymph nodes metastasis

Negative

Positive

TNM stage
$33(45)$

$40(55)$

$38(52)$

35 (48)

$51(49)$

$52(51)$

0.837

$14(19)$

$59(81)$

$9(12)$

$5(5)$

28 (39)

$10(10)$

$36(49)$

$88(85)$

$<0.001$

$56(77)$

$62(63)$

$17(23)$

$41(37)$

0.022 


\begin{tabular}{lccc}
\hline II & $21(29)$ & $46(45)$ & \\
III & $11(15)$ & $31(30)$ & $<0.001$ \\
IV & $7(9)$ & $11(11)$ & \\
Growth pattern & $29(39)$ & $40(37)$ & 0.878 \\
Expansive & $44(61)$ & $63(63)$ & \\
Infiltrative & & & \\
Differentiation & $60(82)$ & $63(61)$ & \\
Well-moderately & $13(19)$ & $40(39)$ & \\
Poorly & & \\
\hline
\end{tabular}


Distant normal mucosa

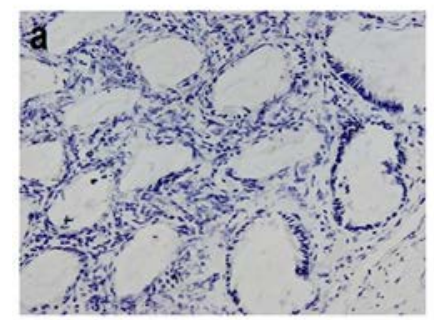

Adenoma

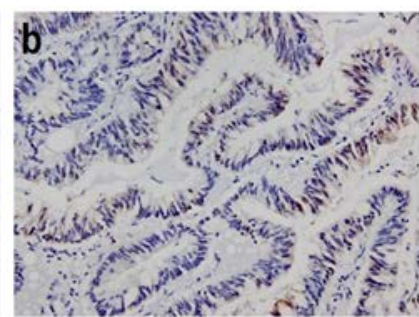

Primary cancer

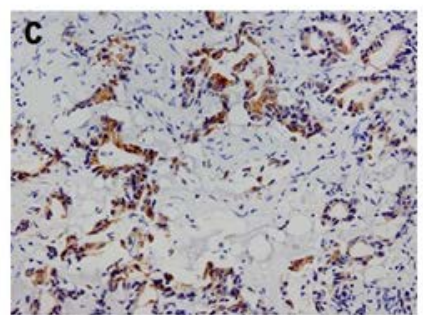

Fig. 1 AKAP10 expression was detected in distal normal mucosa, adenoma, and primary cancer by immunohistochemical analysis. (a) Negative expression in distal normal mucosa, positive expression in (b) tubular adenoma and (c) primary cancer with moderated differentiated. All images were taken at a magnification of $\times 400$.
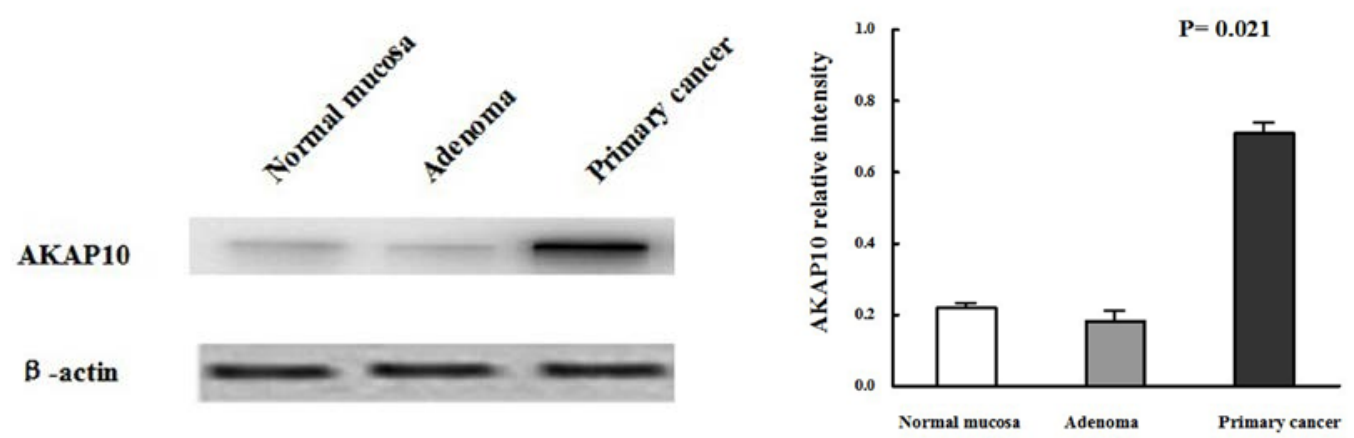

Fig. 2 Western blot assay showed that (a) the expression of AKAP10 was abundantly increased in the primary cancer than adenoma or distant normal mucosa and (b) the quantitative analysis confirmed this alteration was in accordance with that of the IHC staining. 


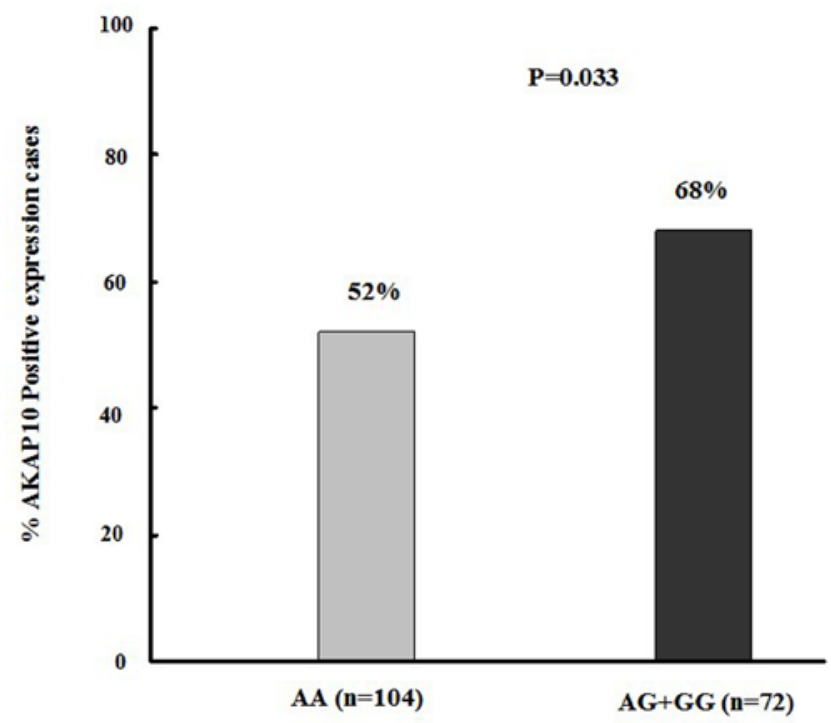

Fig. 3 The positive expression of AKAP10 significantly increased in colorectal cancer patients with the variant (AG+GG) genotypes (68\%), compared with AA genotype (52\%). 